\title{
LA INTENSIDAD DE LLUVIA EN ENTORNOS MEDITERRÁNEOS. VALORES EXTREMOS SEGÚN LA ESCALA DE OBSERVACIÓN
}

\author{
Ana M. Camarasa-Belmonte \\ J. Soriano \\ Departamento de Geografía. Universidad de Valencia \\ ana.camarasa@uv.es, jusogar@alumni.uv.es
}

\section{RESUMEN}

Este trabajo estudia la estructura de las intensidades máximas de lluvia a diferentes escalas de observación, entre los 5 minutos y las 24 horas, en la Confederación Hidrográfica del Júcar, en base a datos del SAIH (1994-2007). Aborda además la influencia de los factores de altitud y distancia al mar, así como de la exposición del territorio: litoral-interior y compartimentación general del relieve. Los resultados muestran una gran dependencia de la intensidad en relación con la escala de observación, una influencia significativa de la distancia al mar y dos patrones de intensidad.

Palabras clave: intensidad de lluvia, Mediterráneo, escala temporal, topografía, distancia al mar.

\section{ABSTRACT}

This paper studies the structure of rainfall intensities at different scales of observation, between 5 minutes and 24 hours, in the Júcar River Division, based on SAIH data (19942007). Also addresses the influence of factors of altitude and distance to the sea, as well

Fecha de recepción: abril 2013.

Fecha de aceptación: diciembre 2013.

Este trabajo ha sido realizado en el marco del proyecto CGL2013-42728-R, financiado por el Ministerio de Ciencia e Innovación y por fondos FEDER. 
as exposure of the territory: coastal-interior and compartmentalization of the territory. The results show a highly rainfall intensity dependence on scale of observation, a significant influence of the distance to the sea and two intensity patterns

Key words: rainfall intensity, Mediterranean climate, time scale, topography, distance to the sea.

\section{INTRODUCCIÓN}

Las lluvias extremas mediterráneas se producen en un contexto climático de tipo semiárido, dominado por sucesos torrenciales de alta energía y baja frecuencia. Se trata de episodios de lluvia de gran intensidad y corta duración, cuya distribución espacio-temporal es tremendamente variable (Romero et al, 1999; Llasat, 2001; Peñarrocha et al., 2002; Armengot, 2002; Beguería et al., 2009). Un ejemplo de ello lo constituye la fachada mediterránea de la Peninsula Ibérica, donde a pesar de que la lluvia media anual oscila entre los 500 y los $700 \mathrm{~mm}$, un único evento puede llegar a duplicar, incluso triplicar esta media (Gil Olcina, 1989), llegando a registrarse records de más de 800 mm en un día (como ocurrió en la ciudad de Gandía, en noviembre de 1987). En general es muy frecuente que en los momentos álgidos de las tormentas se superen los $100 \mathrm{~mm} / \mathrm{h}$, llegándose incluso a registrar intensidades puntuales de más de $375 \mathrm{~mm} / \mathrm{h}$.

Como la mayor parte de estas lluvias son de origen convectivo, concentran gran cantidad de precipitación en muy poco tiempo y presentan una gran heterogeneidad espacial. Sirva como ejemplo el hietograma registrado en Portaceli (Valencia) durante los días 8-9 de septiembre de 1990, donde precipitaron 152 mm en 15h 30', de los cuales $143.6 \mathrm{~mm}$ (18\% del total) se concentraron en apenas 3 horas (18\% del tiempo de duración de la tormenta) y sobre una superficie que no superaba los $15 \mathrm{~km}^{2}$ de extensión.

En consecuencia, el parámetro crítico de estos eventos no es tanto la cantidad de lluvia que pueden acumular, sino la intensidad que pueden alcanzar. Magnitudes como las arriba descritas resultan determinantes en la dinámica de erosión del suelo (Poesen and Bunte, 1996, Larson et al., 1997, Martínez-Mena et al., 2001); en el desencadenamiento de debrisflow (Cannon, 1988; Wilson and Wieczorek, 1995; Bacchini and Zannoni, 2003; ChienYuan et al., 2005) y, sobre todo, en hidrología, ya que afectan a los procesos de conversión lluvia-caudal (Greenbaum et al., 1998; Bull et al., 1999; Yair and Raz-Yassif, 2004). En este sentido, para el caso particular de las ramblas está demostrado que las grandes intensidades de lluvia pueden reducir significativamente la capacidad inicial de infiltración del suelo y los umbrales de escorrentía, de manera que se produce circulación superficial incluso en suelos que aún no se han saturado (Yair and Lavee, 1985; Bull et al., 1999; Lange et al., 2003; Cammeraat, 2004). La mayoría de las avenidas súbitas mediterráneas (flash-flood) son muy dependientes de la lluvia y tienen su origen en estos sucesos de gran intensidad (Kokknen et al., 2004; Cudennec et al., 2007; Braken et al., 2008).

A pesar de la importancia del parámetro de intensidad de lluvia, no resulta fácil identificar unos umbrales de referencia (Montesarchio et al, 2009; Zehe y Sivapalan, 2009) porque la estructura de la lluvia difiere según la escala de observación utilizada (Waymire y Gupta, 1981; Valdés et al., 1985; Jebari et al., 2007; Dunkerley, 2008; Berne et al., 2009), de 
manera que a medida que reducimos el intervalo de tiempo crecen los valores de intensidad e irregularidad de la precipitación. No está claro, por tanto, cuál es el intervalo temporal más apropiado para medir la intensidad.

El estudio de células convectivas aconseja trabajar con umbrales de intensidad entorno a los 48-50 mm/h y un período aproximado de una hora entre un evento y otro (Llasat, 2001). Algunos estudios de debris-flow utiliza la lluvia acumulada durante los 14 días precedentes al suceso y la intensidad horaria de los 5 días anteriores al inicio del movimiento (ChienYuan et al., 2005). Por lo que respecta a la pérdida de suelo, Martinez-Mena et al. (2001) consideran que una intensidad por encima de los $15 \mathrm{~mm} / \mathrm{h}$ durante 30 minutos puede ser considerada como «lluvia erosiva». En cuanto a la producción de escorrentía en ramblas, se manejan umbrales entorno a los 30-60 mm acumulados en varias horas (Camarasa y Segura, 2001; Cammeraat, 2004).

El estudio de intensidades máximas debería partir de intervalos de observación a escala minutal. No obstante, en la mayoría de los países mediterráneos, los datos de lluvia se registran cada 24 horas, escala claramente insuficiente. Por ello muchos trabajos utilizan modelos matemáticos que desagregan la lluvia en intervalos más finos mediante la transformación de la información diaria (Salson-Casado and García-Bartual, 1998; Bacchini and Zannoni, 2003; Egozcue et al., 2006; Rusjan et al., 2009). Estudios recientes, no obstante, han demostrado que los valores estimados por estos métodos difieren bastante de la realidad (Bengtsson and Milloti, 2010).

Resulta, por tanto, esencial estudiar la estructura de la intensidad a diferentes escalas. En España aproximaciones de estas características se están llevando a cabo a partir de la red SAIH (Sistema Automático de Información Hidrológica), que provee datos de intensidad de lluvia en tiempo real y cada cinco minutos. Esta red, operativa de 1989, ha permitido realizar un seguimiento empírico del diferente comportamiento de la intensidad de lluvia según la escala temporal de observación. Una vez constatado el hecho de que la estructura de la intensidad de lluvia cambia según la escala temporal de observación (Camarasa et al, 2010) surgen nuevas preguntas: ¿cuáles son las escalas de observación más representativas?; ¿cuál es la estructura de los valores extremos de intensidad para diferentes escalas temporales de observación?; ¿qué factores geográficos están condicionando los valores extremos?; ¿difiere el papel que juegan estos factores de unas escalas de observación a otras?.

Intentando contestar a estas cuestiones, el presente artículo analiza el comportamiento de las intensidades máximas de lluvia en la Confederación Hidrográfica del Júcar, para diferentes escalas de observación, desde los cinco minutos a las 24 horas, durante un período de catorce años de registro continuado (1994-2007). Aborda, además, la influencia de los factores geográficos de altitud y distancia al mar para las distintas escalas temporales, así como la exposición del territorio según diferentes criterios: litoral-interior y compartimentación general del relieve.

\section{II. ÁREA DE ESTUDIO Y DATOS}

El area de studio (figura 1) cubre el ámbito de la Confederación Hidrográfica del Júcar $\left(42.989 \mathrm{~km}^{2}\right)$. Situada en el levante peninsular, manifiesta una gran diversidad geográfica. Desde el punto de vista geomorfológico se distinguen tres unidades principales: (1) los sis- 
temas montañosos, (2) la plataforma meseteña y (3) la llanura costera. El ámbito montañoso está constituido por dos grandes alienaciones (la septentrional, de dirección ibérica NW-SE y la meridional, de dirección bética SW-NE). El sector occidental está constituido por la plataforma meseteña y el sector oriental por una gran llanura aluvial de más de $400 \mathrm{~km}$ de longitud y hasta $40 \mathrm{~km}$ de anchura en las zonas más amplias (Estrela et al., 2004). Desde un punto de vista climático la zona, ubicada entre los $38^{\circ}$ y los $40^{\circ} \mathrm{N}$, presenta un clima típico mediterráneo. La media anual de precipitación es de unos $500 \mathrm{~mm}$ aproximadamente, con gradientes que van desde los $300 \mathrm{~mm}$ en el sector meridional hasta más de 800 en el sector septentrional. Las mayores intensidades se registran con ocasión de fuertes episodios que se concentran en otoño, con un segundo máximo en primavera.

Nos encontramos, por tanto, ante un territorio muy heterogéneo, que presenta una marcada dicotomía interior-costa, con grandes alineaciones montañosas que lo compartimenta (favoreciendo la entrada de flujos húmedos en algunos sectores e inhibiéndolos en otros) y con un clima mediterráneo propenso a la generación de lluvias muy intensas, concentradas en el tiempo y con un patrón muy variable en el espacio.

Por lo respecta a los datos de intensidad de lluvia, se partió de la información cincominutal, suministrada por la red SAIH-Júcar, a partir de 147 pluviómetros. El período de estudio abarca catorce años, desde 1994 a 2007, de manera continuada. Llegados a este punto cabe aclarar, que si bien el SAIH-Júcar comienza a proporcionar datos en 1989, no existe un registro continuo fiable hasta 1994 (tras el cambio del sistema de adquisición de datos). La instalación de esta red ha supuesto un gran cambio cualitativo respecto a la red tradicional de pluviómetros. Por una parte, la reducción del tiempo de observación a cinco minutos ha permitido identificar la verdadera estructura interna de las precipitaciones, ya que con el dato diario aparecía enmascarada, sobre todo si tenemos en cuenta que las mayores intensidades las originan células convectivas de escasa duración temporal. Por otra parte el funcionamiento en tiempo real del sistema ha supuesto un gran avance en la toma de decisiones dentro del marco de la gestión hídrica en tiempo real.

En cuanto al análisis de la influencia de los factores geográficos en las intensidades máximas, el estudio se basa en los datos altitud y distancia lineal al mar de los puntos donde están localizados los pluviómetros. Asimismo se trabajará con diferentes escenarios de exposición del territorio a los flujos dominantes, en función de dos criterios: la dicotomía litoral-interior y la compartimentación del territorio según las grandes estructuras del relieve.

Para ello, y dada la heterogeneidad espacial del área de estudio, se partió de 11 unidades superficiales (denominadas zonas) que, aunque presentan diferente extensión, conforman áreas geográficas homogéneas. Estas unidades se fueron reagrupando para conformar unidades mayores o escenarios, que obedecían a diferentes criterios de exposición: litoralinterior y compartimentación del relieve). Aunque en la bibliografía se pueden encontrar gran número de métodos, cuantitativos y cualitativos, para establecer unidades espaciales homogéneas (Gabriele and Arnell, 1991; Brunetti et al., 2001; Kysely et al., 2007), en este trabajo se parte de las establecidas por la Confederación Hidrográfica del Júcar. Se siguen criterios mixtos topográficos e hidrológicos (unidades topográficas de cierta entidad y subcuencas de los grandes colectores). Dada la escasez de pluviométros, la aplicación de métodos automáticos podría generar unidades espaciales demasiado dependientes de los datos de lluvia, incompatibles con la geografía subyacente. Las zonas con las que se trabaja atienden 
a criterios cualitativos e interpretativos, que respetan las grandes divisorias de aguas del territorio, así como los sectores de drenaje más importantes. La agrupación de estas zonas para conformar unidades mayores o escenarios lleva implícita la consideración de la geografía física del territorio.

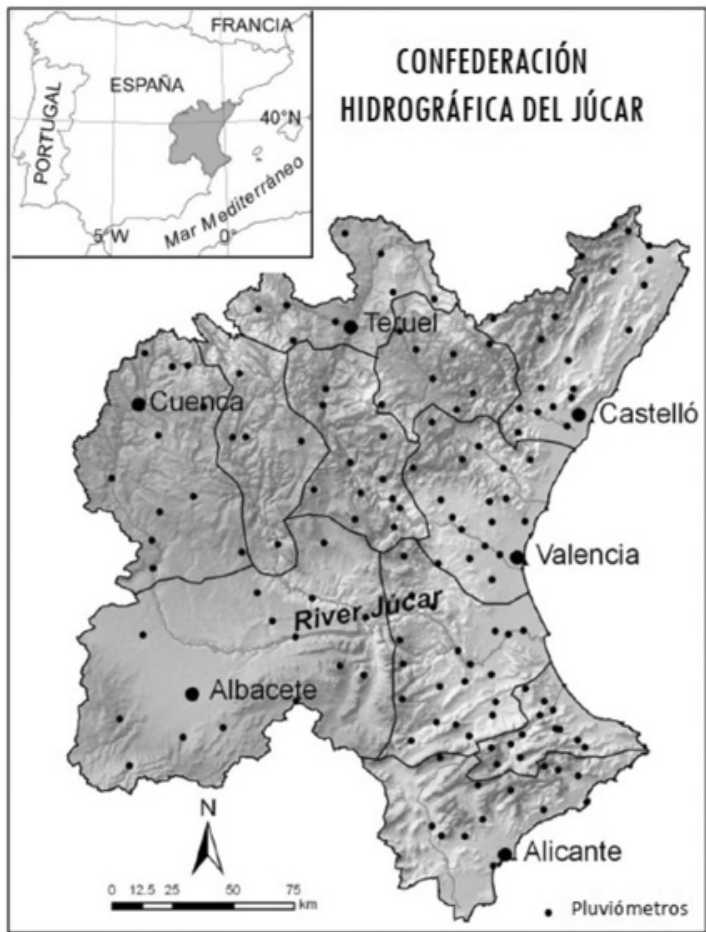

\section{DISTRIBUCIÓN DE LAS INTENSIDADES MÁXIMAS PARA CADA ESCALA TEMPORAL DE OBSERVACIÓN}

\section{III.1. Selección de las escalas más representativas}

Con objeto de reconocer el comportamiento de la intensidad de lluvia en cada escala temporal, los datos de lluvia, originalmente registrados cada 5 minutos, fueron filtrados y reescalados, mediante medias móviles, a intervalos de 15 minutos, 30 minutos, $1 \mathrm{~h}, 2 \mathrm{~h}, 3 \mathrm{~h}, 4 \mathrm{~h}$, $6 \mathrm{~h}, 12 \mathrm{~h}$ y $24 \mathrm{~h}$. Se obtuvieron los valores máximos de intensidad (en $\mathrm{mm} / \mathrm{h}$ ) en cada punto de medición para cada una de las escalas. A partir de aquí se trabajó con dos indicadores, por una parte el valor máximo absoluto registrado para cada intervalo (como techo de referencia) y, por otra, la media de las máximas registradas en los 147 puntos (para disponer de una medida más robusta). 
La figura 2 muestra las curvas de tendencia de ambos parámetros, con coeficientes de determinación $\mathrm{r}^{2}$ superiores a 0.9 , lo que pone de manifiesto que la intensidad de lluvia es claramente dependiente de la escala de observación. Ambos indicadores muestran dos puntos de inflexión en los intervalos de $1 \mathrm{~h}$ y $6 \mathrm{~h}$. Se diferencian así, tres tramos de tendencia: a) un primer tramo entre los 5 minutos y la hora, con las intensidades más altas; b) un tramo intermedio entre $1 \mathrm{~h}$ y $6 \mathrm{~h}$ y c) un tramo final entre las $6 \mathrm{~h}$ y las 24 h, con las intensidades más bajas.

Según se desprende del análisis anterior, existen cuatro intervalos temporales de observación que resultan representativos: 5 minutos, 1 hora, 6 horas y 24 horas. A partir de aquí, el análisis se basará en estas cuatro escalas temporales principales más tres intervalos de apoyo (15 minutos, 30 minutos y 12 horas). No se analizarán los datos correspondientes a los intervalos de 2 horas, 3 horas y 4 horas por considerarlos redundantes.



\section{III.2. Distribución espacial de las intensidades máximas para cada escala de observación}

Las curvas de tendencia de la figura 2, además de demostrar la dependencia de las intensidades máximas de lluvia respecto del tiempo, parecen indicar una estructura diferente de las mismas en función del intervalo de observación. Con objeto de comprobar la viabilidad de esta hipótesis se han llevado a cabo análisis de distribuciones de frecuencias de las intensidades máximas de lluvia para los intervalos de tiempo seleccionados y su posterior contraste mediante pruebas estadísticas de comparación de medias.

La figura 3 y la tabla 1 muestran los valores estadísticos de las distribuciones, para un 95\% de intervalo de confianza. Las pruebas de normalidad llevadas a cabo mediante el test de Kolmogorov-Smirnov ponen de manifiesto que las intensidades máximas registradas a intervalos temporales de $5 \mathrm{~min} ., 15 \mathrm{~min} ., 30 \mathrm{~min}$. y $1 \mathrm{~h}$ presentan distribuciones normales, mientras que para el resto de intervalos $(6 \mathrm{~h}, 12 \mathrm{~h}$ y $24 \mathrm{~h})$ las distribuciones no son normales. 
Por lo que respecta a los estadísticos de forma, las intensidades registradas en los intervalos de tiempo comprendidos entre 5 minutos y 1 hora presentan distribuciones simétricas y mesocúrticas, mientras que para intervalos de tiempo mayores muestran asimetrías positivas y son leptocúrticas, destacando el intervalo de las 6 horas como el que presenta mayor asimetría y curtosis. Todo ello pone de manifiesto que los valores medios de las intensidades máximas resultan representativos para el caso de las escalas temporales más pequeñas, desde 5 minutos hasta una hora, mientras que a partir de la hora, la concentración de valores es mucho menor, con una mayor frecuencia de los valores por debajo de la media. El intervalo de 6 horas constituye un punto de inflexión en el comportamiento de las intensidades máximas hacia una reducción de la intensidad, ya que presenta la mayor frecuencia de valores por debajo de su media y con una mayor dispersión respecto de la misma.

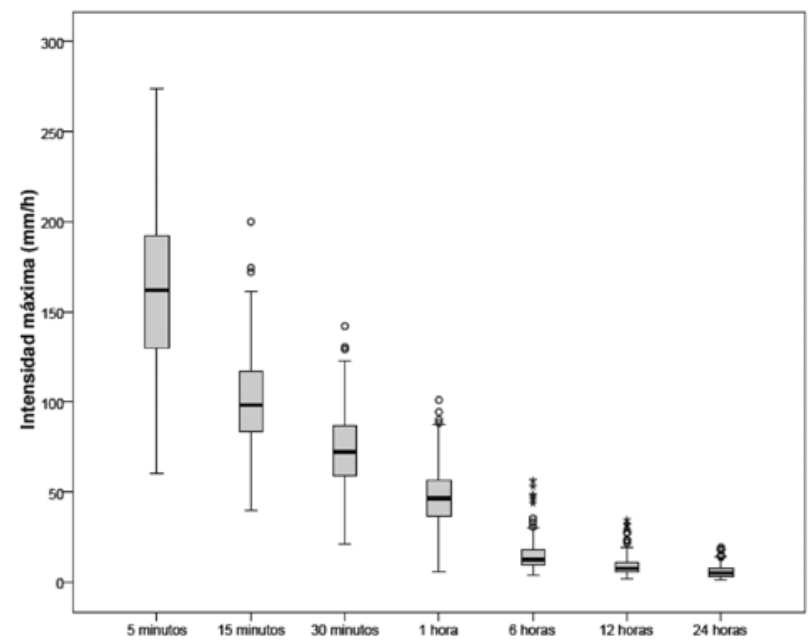

Tabla 1

CARACTERÍSTICAS DE LAS INTENSIDADES MÁXIMAS DE LLUVIA PARA CADA ESCALA DE OBSERVACIÓN

\begin{tabular}{|c|c|c|c|c|c|c|c|c|c|c|c|c|c|c|}
\hline \multirow{2}{*}{$\begin{array}{l}\text { Intervalo de } \\
\text { observación }\end{array}$} & \multicolumn{2}{|c|}{$\begin{array}{l}\text { Media } \\
(\mathrm{mm} / \mathrm{h})\end{array}$} & \multirow{2}{*}{$\begin{array}{c}\text { Desviación } \\
\text { estándar }\end{array}$} & \multirow{2}{*}{$\begin{array}{l}\text { Min. } \\
(\mathrm{mm} / \mathrm{h})\end{array}$} & \multirow{2}{*}{$\begin{array}{c}\text { Max. } \\
(\mathrm{mm} / \mathrm{h})\end{array}$} & \multicolumn{2}{|c|}{ Asimetría } & \multicolumn{2}{|c|}{ Curtosis } & \multicolumn{4}{|c|}{ Cuartiles } & \multirow{2}{*}{\begin{tabular}{|c|} 
Test \\
K-S \\
$\mathrm{p}$ \\
\end{tabular}} \\
\hline & Valor & Error & & & & Valor & Error & Valor & Error & 25 & 50 & 75 & 100 & \\
\hline 5 minutos & 163 & 3,7 & 45,2 & 60 & 273,6 & 0,18 & 0,2 & $-0,24$ & 0,4 & $<129$ & 129-162 & $162-192$ & $>192$ & 0,85 \\
\hline 15 minutos & 102,2 & 2,3 & 29 & 40 & 200 & 0,51 & 0,2 & 0,44 & 0,4 & $<83$ & $83-98$ & $98-117$ & $>117$ & 0,5 \\
\hline 30 minutos & 73,1 & 1,8 & 22 & 20,8 & 142 & 0,45 & 0,2 & 0,27 & 0,4 & $<59$ & $59-72$ & $72-86,7$ & $>86,7$ & 0,62 \\
\hline 1 hora & 48,5 & 1,4 & 17,3 & 5,6 & 101 & 0,64 & 0,2 & 0,41 & 0,4 & $<36$ & $36-46$ & $46-56,7$ & $>56,7$ & \begin{tabular}{|l|}
0,07 \\
\end{tabular} \\
\hline 6 horas & 14,9 & 0,7 & 9,2 & 3,6 & 56,3 & 2,2 & 0,2 & 6,3 & 0,4 & $<9$ & $9-12$ & $12-17,6$ & $>17,6$ & 0,002 \\
\hline 12 horas & 9,3 & 0,5 & 6,1 & 1,9 & 34,5 & 1,9 & 0,2 & 4,5 & 0,4 & $<5$ & $5-7,7$ & $7,7-11$ & $>11$ & 0,001 \\
\hline 24 horas & 5,8 & 0,3 & 3,7 & 1,3 & 19,2 & 1,5 & 0,2 & 2,2 & 0,4 & $<3$ & $3-4,8$ & $4,8-7,4$ & $>7,4$ & 0,002 \\
\hline
\end{tabular}


Con objeto de confirmar que realmente las intensidades máximas presentan diferente estructura según la escala temporal de observación, se aplicaron pruebas estadísticas de comparación de medias, de tipo paramétrico (prueba T para muestras relacionadas) para las distribuciones normales (intervalos de $5 \mathrm{~min} ., 15 \mathrm{~min} ., 30 \mathrm{~min}$. y $1 \mathrm{~h}$ ) y de tipo no paramétrico (prueba de Wilcoxon) para el resto de intervalos horarios (6h, $12 \mathrm{~h}$ y $24 \mathrm{~h}$ ), de distribución no normal. Los resultados ponen de manifiesto, con un $95 \%$ de confianza, que existen diferencias significativas entre las muestras analizadas, lo que indica que efectivamente las intensidades máximas de lluvia presentan una estructura diferente para cada intervalo temporal de observación.

Figura 4

CARTOGRAFÍA DE INTENSIDADES MÁXIMAS, AGRUPADAS POR CUARTILES, PARA INTERVALOS DE OBSERVACIÓN DE 5 minutos $\left(Q_{25^{\circ}}:<129 \mathrm{~mm} / \mathrm{h}, Q_{50^{*}}: 129-162 \mathrm{~mm} / \mathrm{h}, Q_{75^{\circ}}: 162-192 \mathrm{~mm} / \mathrm{h} ; \mathrm{Q}_{100^{\circ}}:>192 \mathrm{~mm} / \mathrm{h}\right) ; 1 \mathrm{HORA}\left(\mathrm{Q}_{25^{\circ}}<36 \mathrm{~mm} / \mathrm{h}, \mathrm{Q}_{50^{\circ}}\right.$ $\left.36-46 \mathrm{~mm} / \mathrm{h}, Q_{75}: 46.4-56.7 \mathrm{~mm} / \mathrm{h}, Q_{100^{\circ}}:>56.7 \mathrm{~mm} / \mathrm{h}\right) ; 6 \mathrm{HORAS}\left(\mathrm{Q}_{25^{\circ}}:<9 \mathrm{~mm} / \mathrm{h}, \mathrm{Q}_{50}: 9-12 \mathrm{~mm} / \mathrm{h}, \mathrm{Q}_{75^{\circ}}: 12-17.6 \mathrm{~mm} / \mathrm{h}, \mathrm{Q}_{100^{\circ}}\right.$ : $>17.6 \mathrm{~mm} / \mathrm{h})$ Y $24 \mathrm{HORAS}\left(Q_{25^{\circ}}:<3 \mathrm{~mm} / \mathrm{h}, Q_{50}: 3-4.8 \mathrm{~mm} / \mathrm{h}, Q_{75^{\circ}}: 4.8-7.4 \mathrm{~mm} / \mathrm{h}, Q_{100}:>7.4 \mathrm{~mm} / \mathrm{h}\right)$

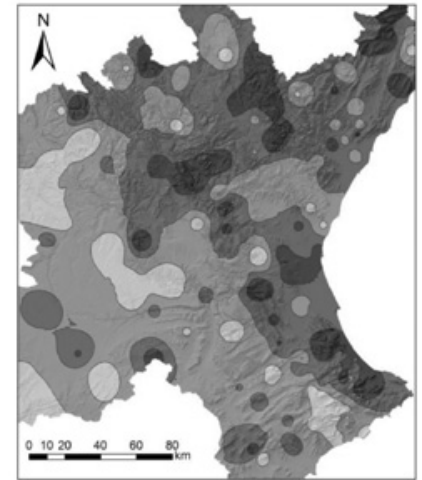

5 minutos

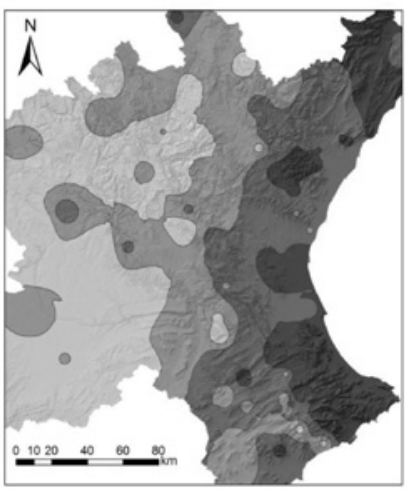

6 horas

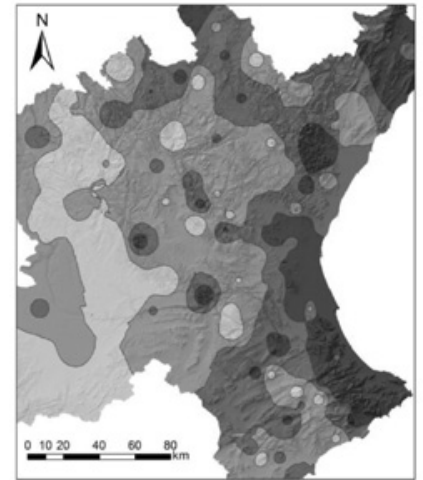

1 hora

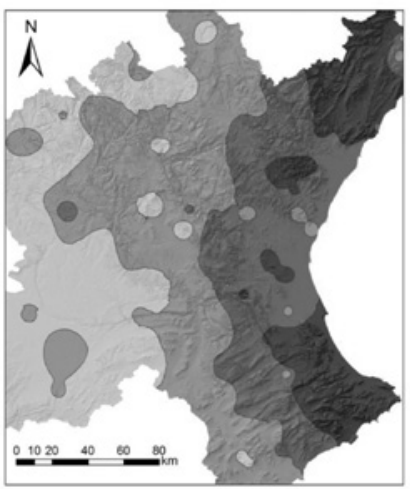

24 horas 
Por último, el análisis de la distribución espacial de las intensidades máximas se abordó a partir de la cartografía de la figura 4, que representa los valores de intensidad, agrupados por cuartiles, para cada intervalo de observación. A medida que incrementa el intervalo temporal se reduce la superficie afectada por los valores más altos de intensidad. En las escalas comprendidas entre los 5 minutos y una hora, la mayor parte del territorio está afectado por rangos de intensidad correspondientes al segundo cuartil (entre $25 \%$ y $50 \%$ ), y afecta principalmente a la franja costera y los relieves del Sistema Ibérico. A partir de las 6 horas, la mayor extensión espacial corresponde a las bajas intensidades del primer cuartil que cubren el interior del territorio, relegándose las mayores intensidades a la franja costera.

En síntesis, las intensidades máximas de lluvia presentan una estructura diferente en función del intervalo temporal con que se registren. Además, su distribución espacial parece estar muy condicionada por la dicotomía costa/interior así como por las grandes cadenas montañosas.

\section{INFLUENCIA DE LOS FACTORES GEOGRÁFICOS DE TOPOGRAFÍA Y DISTANCIA AL MAR}

Los valores máximos de intensidad con los que estamos trabajando son consecuencia de situaciones de gran inestabilidad meteorológica potenciada por factores locales, tales como la topografía y la distancia al mar. (Millán et al., 1995; Jebari et al., 2007; Allamano et al., 2009; Boni et al., 2005). Somos conscientes de la enorme importancia que para el análisis de episodios torrenciales reviste el estudio de las condiciones atmosféricas que los han desencadenado (Martín Vide, 1987; Romero et al., 1999; Peñarrocha et al., 2002; Homar et al., 2002; Ziv et al., 2004). En el presente trabajo, sin embargo, centraremos nuestro análisis en la influencia de los factores geográficos de topografía y distancia al mar, ya que no estamos trabajando sobre episodios concretos, sino sobre las intensidades máximas registradas en cada intervalo temporal, independientemente del evento al que pertenezcan. En este sentido, tales factores geográficos ejercen una influencia constante sobre los procesos de precipitación. En algunos casos sólo actúan como modificadores y en otros (especialmente para intervalos inferiores a 1 h) como desencadenantes (Frei and Schaär, 1998; Houze et al. 2001; Roe, 2005; Boni et al., 2008)

Con objeto de analizar el tipo de relación entre las intensidades máximas para diferentes intervalos de observación y los factores geográficos se ha llevado a cabo un estudio multivariante que ha consistido, por una parte en un análisis de regresión múltiple entre las intensidades máximas y las variables independientes de altitud y distancia al mar, y, por otra parte, en un análisis cluster de dichas variables. Mediante la regresión múltiple no se pretende llegar a construir un modelo de predicción de lluvia, sino únicamente explorar qué variables resultan más significativas y qué tipo de relación se ajusta mejor a la intensidad de lluvia, para cada resolución temporal. Mediante el análisis cluster se busca detectar si, para dichas resoluciones temporales, existen patrones de comportamiento distintos de la precipitación respecto de los factores geográficos.

El análisis de regresión múltiple es de tipo lineal, sin embargo se ha comprobado que la relación entre las variables es, en algunas ocasiones, de tipo lineal y, en otras, de tipo logarítmico ó semi-logarítmico. La figura 5 constituye un ejemplo dependencia entre las intensidades máximas y la distancia al mar para los intervalos temporales de 30 minutos y de 24 horas. 
En ambos casos la relación resulta significativa para un intervalo de confianza del 95\%, sin embargo para la escala de 30 minutos es de tipo lineal, mientras que para 24 horas es de tipo semi-logarítmico. Por ello, con objeto de contemplar las posibles componentes no lineales, una tarea preliminar ha consistido en transformar las variables en logarítmicas y determinar qué tipo de relación presentaba los mayores ajustes para cada intervalo temporal.

Figura 5

TIPOS DE RELACIÓN ESTADÍSTICA ENTRE LA DISTANCIAAL MAR Y LA INTENSIDAD DE LLUVIA:

(A) LINEAL PARA 30 MINUTOS Y (B) SEMILOGARÍTMICA PARA 24 HORAS
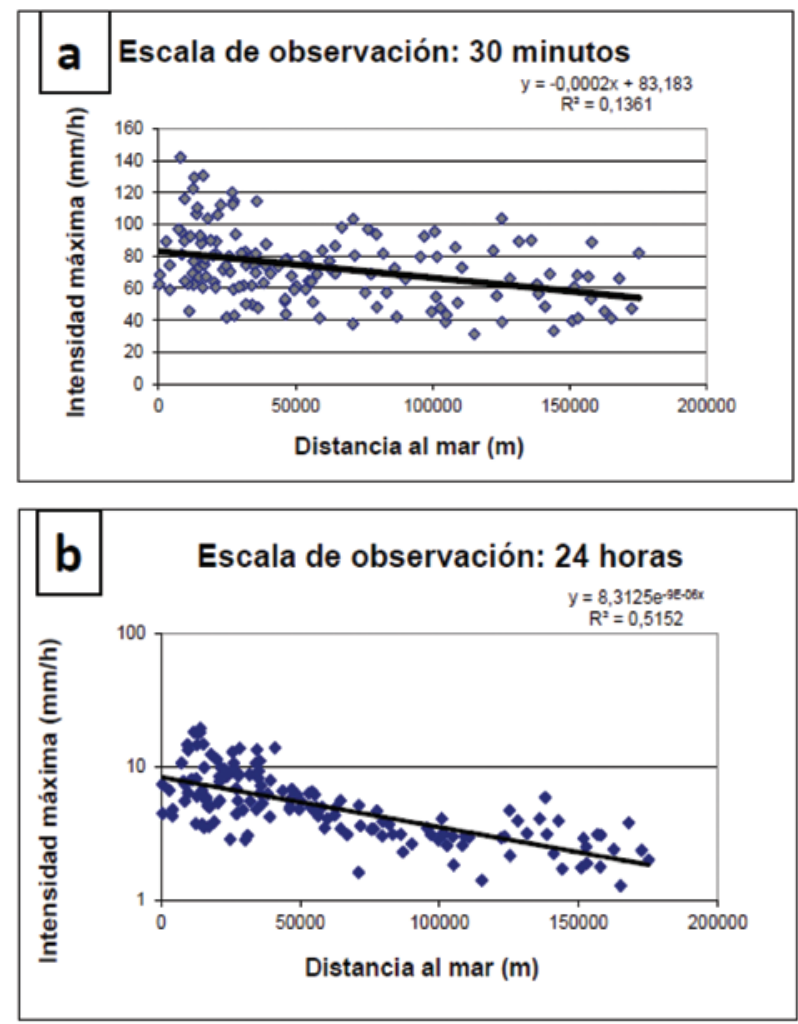

La tabla 2 muestra los resultados de la regresión múltiple. Lo primero que puede observarse es cómo, en función del intervalo temporal, varía el tipo de relación entre las variables independientes y las dependientes, pasando de un tipo lineal entre los 5 minutos y 1 hora, a un tipo semi-logarítmico para los intervalos comprendidos entre 6 horas y 24 horas. Por lo que respecta a la significación de las variables explicativas, destaca la distancia al mar, que resulta significativa, para casi todos los intervalos temporales (con excepción de los 5 minutos), especialmente para las 12 horas y las 24 horas, en que llega a explicar el $50 \%$ de los casos. En cuanto al factor de altitud, resulta significativo entre los 30 minutos y las 6 horas. 
Tabla 2

RESULTADOS DEL ANÁLISIS DE REGRESIÓN ENTRE LA INTENSIDAD DE LLUVIA Y LOS

FACTORES GEOGRÁFICOS DE DISTANCIAAL MAR YALTITUD

\begin{tabular}{|c|c|c|c|c|c|c|}
\hline \multirow[b]{2}{*}{$\begin{array}{l}\text { Intervalo de } \\
\text { observación }\end{array}$} & \multirow[b]{2}{*}{$\begin{array}{l}\text { Tipo de } \\
\text { relación }\end{array}$} & \multirow[b]{2}{*}{$\mathbf{R}^{2}$} & \multicolumn{2}{|c|}{ Distancia al mar } & \multicolumn{2}{|c|}{ Altitud } \\
\hline & & & $\begin{array}{l}\text { Significación para } \\
\text { un intervalo de } \\
\text { confianza del } 95 \% \\
\text { (p) }\end{array}$ & $\begin{array}{c}\text { Coeficiente } \\
\text { B }\end{array}$ & $\begin{array}{l}\text { Significación para } \\
\text { un intervalo de } \\
\text { confianza del } 95 \% \\
\text { (p) }\end{array}$ & Coeficiente B \\
\hline 5 minutos & Lineal & 0.02 & NO (0.108) & $-1.58 \mathrm{E}-4$ & NO (0.882) & 0.002 \\
\hline 15 minutos & Lineal & 0.10 & SÍ (0.014) & $-1.48 \mathrm{E}-4$ & NO $(0.380)$ & -0.006 \\
\hline 30 minutos & Lineal & 0.17 & SÍ (0.010) & $-1.13 \mathrm{E}-4$ & SÍ (0.037) & -0.010 \\
\hline 1 hora & Lineal & 0.23 & SÍ (0.002) & $-1.044 \mathrm{E}-4$ & SÍ (0.017) & -0.009 \\
\hline 6 horas & Logarítmica & 0.45 & SÍ $(0.000)$ & $-2.607 \mathrm{E}-6$ & SÍ (0.039) & $-8.851 \mathrm{E}-5$ \\
\hline 12 horas & Logarítmica & 0.50 & SÍ $(0.000)$ & $-3.316 \mathrm{E}-6$ & NO (0.247) & $-4.930 \mathrm{E}-5$ \\
\hline 24 horas & Logarítmica & 0.51 & SÍ (0.000) & $-3.744 \mathrm{E}-6$ & NO (0.986) & $7.728 \mathrm{E}-7$ \\
\hline
\end{tabular}

En general, los valores del coeficiente de determinación $\mathrm{r}^{2}$ son bastante bajos, sobre todo para las escalas temporales más pequeñas. Esto es debido a que las principales variables explicativas están en relación con los factores climáticos desencadenantes de los eventos y no con los factores geográficos que, como ya se ha mencionado, únicamente actúan como potenciadores de las precipitaciones extremas. Para los intervalos temporales inferiores a 1 hora, el efecto de estos factores, aunque resulta estadísticamente significativo, es muy pequeño $\left(\mathrm{r}^{2}<0.2\right)$. A partir de las 6 horas, cobra importancia la influencia de la distancia al mar y de la altitud, tal y como lo demuestra el incremento de los valores de $\mathrm{r}^{2}$ que ahora oscilan entorno al 0.5.

El análisis cluster se llevó a cabo, como ya se ha mencionado, con objeto de comprobar si, para diferentes escalas de observación, existen patrones de comportamiento distintos de la intensidad de lluvia respecto de los factores geográficos.

Los resultados del cluster (tabla 3 ) ponen de manifiesto que, para un intervalo de confianza del 95\%, existen dos patrones diferenciados de la intensidad de lluvia, detectables en todas las escalas temporales de observación. El primer comportamiento agrupa las intensidades más altas de cada escala, asociadas a un rango de altitud que, a excepción de los intervalos de 15 minutos y 12 horas, oscila en un rango de altitud media entre los $250 \mathrm{~m}$ y los $363 \mathrm{~m}$ y a una distancia al mar entre los $21 \mathrm{~km}$ y los $27 \mathrm{~km}$. Los intervalos de 15 minutos y 12 horas constituyen una excepción importante, ya que presentan altitudes medias en torno a los $642 \mathrm{~m}$ y una distancia al mar de 59,7 km. Esta componente describe que las principales intensidades de la mayor parte de los intervalos se alcanzan en una franja de altitud no muy elevada y relativamente cercana al mar. Una posible interpretación de este hecho radica en que, en la zona de estudio, la mayor parte de los aguaceros intensos aparecen ligados a vientos de componente Este provenientes del Mediterráneo (Martín Vide, 1987; Albentosa, 1989; Quereda Sala, 1989; Romero et al., 1999; Peñarrocha et al., 2002; Millán et al., 2004), que una vez penetran en el continente se ven obligados a ascen- 


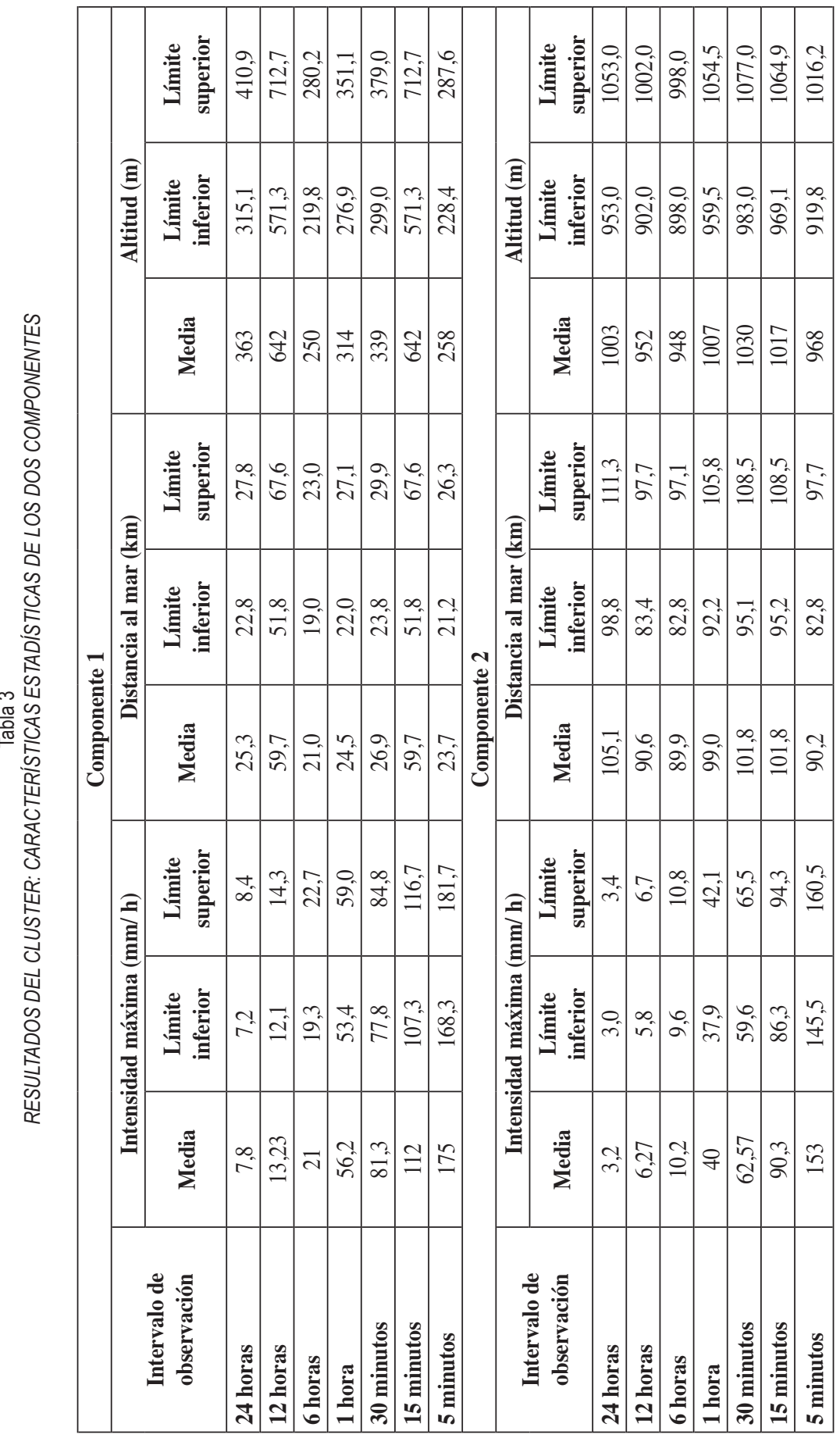


der y a precipitar, en las primeras alineaciones montañosas, relativamente cercanas al mar. De una manera generalizada, estos frentes de disparo se encontrarían entre los 200 y los 400 metros y a una distancia del mar entre 20 y $30 \mathrm{~km}$. Para los intervalos de 15 minutos y 12 horas los rangos de altitud y de distancia al mar son mayores (entre 500 y $700 \mathrm{~m} \mathrm{de}$ altitud y entre 50 y $70 \mathrm{~km}$ del mar).

Por lo que respecta al rol de las primeras alineaciones montañosas como disparador de las mayores inestabilidades frente a vientos húmedos de levante ya fue señalado por Camarasa y Segura (2001) que hablaban de «dos maneras de llover», por debajo y por encima de los 200 $\mathrm{m}$ de altitud, donde las mayores cantidades de lluvia se acumulaban en el retablo montañoso, mientras que las mayores intensidades se registraban en la costa. Con objeto de localizar mejor estos frentes de disparo orográfico se ha estimado la media de las intensidades máximas cada doscientos metros de altitud. Los resultados demuestran que entre los intervalos temporales de cinco minutos y una hora los frentes de disparo están por debajo de los 200 metros, mientras que a partir de la hora, se ubican entre 200 y 400 metros.

Por lo que respecta al segundo comportamiento que se refleja en el análisis cluster, éste es mucho más regular y agrupa las intensidades menores de cada escala temporal, asociadas a altitudes medias que oscilan entre 948 y $1030 \mathrm{~m}$ y distancias medias al mar entre $90 \mathrm{~km}$ y $105 \mathrm{~km}$. Este componente estaría relacionado con un tipo de lluvia más generalizada y continua en el espacio, de mayor frecuencia y menor intensidad que puede responder a aguaceros de tipo frontal y componente $\mathrm{W}$.

\section{LAEXPOSICIÓN}

La relación entre los factores geográficos y las intensidades máximas, que se apunta de manera general para toda el área de estudio, podría estar matizada por la configuración del territorio, en función, por ejemplo, de la dicotomía litoral-interior o de las grandes estructuras del relieve. En este sentido, la subdivisión del área de estudio en zonas (ver apartado dos) permite configurar diferentes escenarios geográficos, mediante su agregación en unidades mayores según criterios de exposición.

Con objeto de comprobar si la configuración general del territorio influía en la relación entre los factores geográficos y las intensidades máximas, se procedió a compartimentar el área de estudio en función de dos criterios: a) la distancia al mar y b) la dirección de las grandes estructuras de relieve (figura 6). En el primer caso se establecieron dos escenarios: 1) zonas próximas a la costa y 2) zonas interiores. En el segundo caso se establecieron cuatro escenarios: 1) zonas que siguen la dirección de las sierras ibéricas NW-SE; 2) zonas que conforman el valle medio-bajo del Río Jucar, de dirección E; 3) zona que sigue la dirección de las sierras béticas expuesta a los vientos de NE y 4) zona que sigue la dirección de las sierras béticas expuesta a los vientos del SE. Como puede verse en la figura 6 , el tamaño de los espacios es muy diferente porque está condicionado por la compleja configuración del relieve. De hecho, el dominio de las sierras béticas se ha subdividido en dos porque la exposición a los vientos del NE o del SE supone importantes diferencias en los totales de lluvia del área. Sobre cada uno de estos espacios se aplicó, como en el apartado anterior, un análisis de regresión múltiple entre las intensidades máximas para cada escala temporal de observación y las variables independientes de distancia al mar y altitud. 
Figura 6

ESCENARIOS DE EXPOSICIÓN: COSTA-INTERIOR Y EN FUNCIÓN DE LA COMPARTIMENTACIÓN DEL RELIEVE

$\AA$

$\AA^{N}$
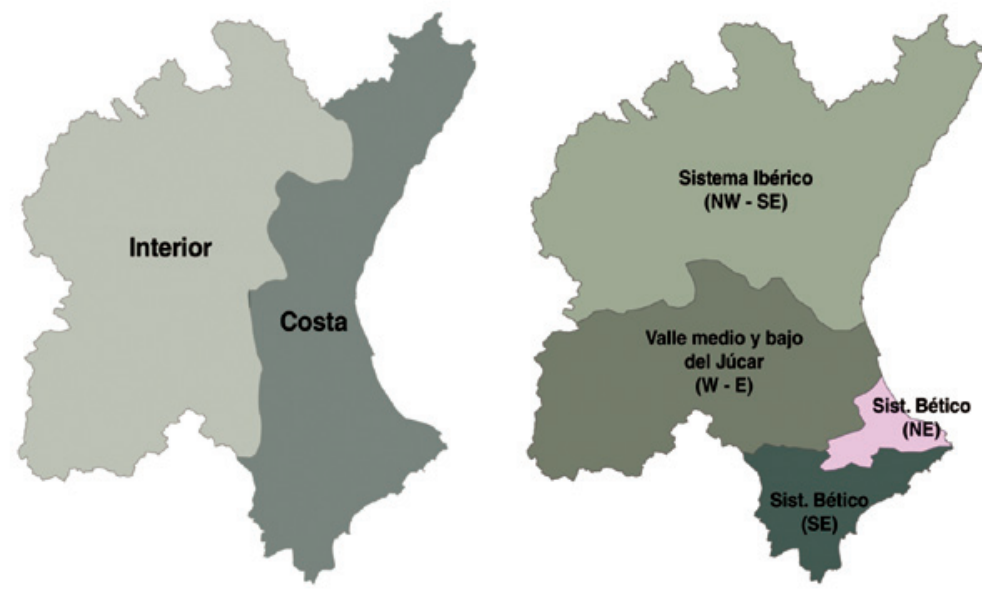

$\stackrel{0}{50} \quad 100 \mathrm{~km}$

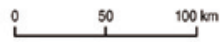

Tabla 4

RESULTADOS DE LA REGRESIÓN ENTRE INTENSIDAD DE LLUVIA Y FACTORES GEOGRÁFICAS PARA LOS ESCENARIOS DE COSTAE INTERIOR

\begin{tabular}{|c|c|c|c|c|c|c|c|}
\hline \multirow[b]{2}{*}{$\begin{array}{c}\text { Escenarios de } \\
\text { exposición }\end{array}$} & \multirow{2}{*}{$\begin{array}{l}\text { Intervalo de } \\
\text { observación }\end{array}$} & \multirow{2}{*}{$\begin{array}{l}\text { Tipo de } \\
\text { relación } \\
\text { estadística }\end{array}$} & \multirow[b]{2}{*}{$R^{2}$} & \multicolumn{2}{|c|}{ Distancia al mar } & \multicolumn{2}{|c|}{ Altitud } \\
\hline & & & & $\begin{array}{c}\text { Significación } \\
\text { para un } 95 \% \text { (p) }\end{array}$ & $\begin{array}{c}\text { Coeficiente } \\
\text { B }\end{array}$ & $\begin{array}{c}\text { Significación } \\
\text { para un } 95 \% \text { (p) }\end{array}$ & $\begin{array}{c}\text { Coeficiente } \\
\text { B }\end{array}$ \\
\hline \multirow{7}{*}{ Costa } & 5 Minutos & Lineal & 0,03 & $\mathrm{NO}(0,723)$ & $-1,282 \mathrm{E}-4$ & NO $(0,259)$ & $-0,180$ \\
\hline & 15 Minutos & Lineal & 0,04 & NO $(0,566)$ & $-1,424 \mathrm{E}-4$ & NO $(0,267)$ & $-0,012$ \\
\hline & 30 Minutos & Lineal & 0,15 & NO $(0,510)$ & $-1,168 \mathrm{E}-4$ & SÍ $(0,016)$ & $-0,019$ \\
\hline & 1 Hora & Semi-log. & 0,19 & $\mathrm{NO}(0,561)$ & $-1,467 \mathrm{E}-6$ & SÍ $(0,003)$ & $-3,318 \mathrm{E}-4$ \\
\hline & 6 Horas & Semi-log. & 0,19 & SÍ $(0.006)$ & $-9,917 \mathrm{E}-6$ & $\mathrm{NO}(0,346)$ & $-1,453 \mathrm{E}-4$ \\
\hline & 12 Horas & Semi-log. & 0,13 & SÍ $(0,006)$ & $-1,049 \mathrm{E}-5$ & $\mathrm{NO}(0,773)$ & $4,466 \mathrm{E}-\mathrm{E}$ \\
\hline & 24 Horas & Semi-log. & 0,10 & Sí $(0,004)$ & $-1,143 \mathrm{E}-5$ & $\mathrm{NO}(0,209)$ & $2,142 \mathrm{E}-4$ \\
\hline \multirow{7}{*}{ Interior } & 5 Minutos & Logarítmica & 0,14 & SÍ $(0,007)$ & $-0,333$ & $\mathrm{NO}(0,147)$ & 0,147 \\
\hline & 15 Minutos & Logarítmica & 0,11 & Sí $(0,032)$ & $-0,239$ & $\mathrm{NO}(0,205)$ & 0,116 \\
\hline & 30 Minutos & Logarítmica & 0,11 & SÍ $(0,038)$ & $-0,255$ & NO $(0,099)$ & 0,169 \\
\hline & 1 Hora & Logarítmica & 0,19 & SÍ $(0,05)$ & $-0,280$ & SÍ $(0,006)$ & $-0,342$ \\
\hline & 6 Horas & Logarítmica & 0,14 & SÍ $(0,008)$ & $-0,337$ & NO $(0,310)$ & 0,106 \\
\hline & 12 Horas & Logarítmica & 0,23 & SÍ $(0,000)$ & $-0,477$ & $\mathrm{NO}(0,710)$ & 0,040 \\
\hline & 24 Horas & Logarítmica & 0,35 & SÍ $(0,000)$ & $-0,595$ & $\mathrm{NO}(0,971)$ & 0,003 \\
\hline
\end{tabular}


Los resultados aparecen en las tablas 4 y 5 . Para el criterio basado en la dicotomía litoralinterior (tabla 4), observamos que, en todas las escalas temporales, las zonas de interior presentan una mayor influencia de los factores geográficos que las de litoral. En general el litoral muestra unos valores de $\mathrm{r}^{2}$ muy bajos, inferiores a 0,2 . La variable «distancia al mar» sólo es significativa en el intervalo de $6 \mathrm{~h}$ y de $24 \mathrm{~h}$, mientras que la altitud lo es para los períodos de $30 \mathrm{~min}$ y $1 \mathrm{~h}$. En este espacio litoral dominan las relaciones semi-logarítmicas. Las zonas de interior, por su parte, presentan relaciones de tipo logarítmico. Destaca la influencia de la variable «distancia al mar» que es significativa para todos los intervalos temporales, alcanzando cierta importancia para las 24 horas, en que llega a explicar el $35 \%$ de los casos. La variable «altitud» únicamente es significativa para el período de 1 hora.

Tabla 5

RESULTADOS DE LA REGRESIÓN ENTRE LA INTENSIDAD DE LLUVIA Y LOS FACTORES GEOGRÁFICOS PARA DIFERENTES ESCENARIOS DE EXPOSICIÓN

\begin{tabular}{|c|c|c|c|c|c|c|c|}
\hline \multirow[b]{2}{*}{$\begin{array}{c}\text { Escenarios } \\
\text { de exposición }\end{array}$} & \multirow[b]{2}{*}{$\begin{array}{l}\text { Intervalo de } \\
\text { observación }\end{array}$} & \multirow{2}{*}{\begin{tabular}{|c|} 
Tipo de \\
relación \\
estadística
\end{tabular}} & \multirow[b]{2}{*}{$\mathbf{R}^{2}$} & \multicolumn{2}{|c|}{ Distancia al mar } & \multicolumn{2}{|c|}{ Altitud } \\
\hline & & & & $\begin{array}{l}\text { Significación } \\
\text { para 95\% (p) }\end{array}$ & $\begin{array}{c}\text { Coeficiente } \\
\text { B }\end{array}$ & $\begin{array}{l}\text { Significación } \\
\text { para 95\% (p) }\end{array}$ & $\begin{array}{c}\text { Coeficiente } \\
\text { B }\end{array}$ \\
\hline \multirow{7}{*}{$\begin{array}{l}\text { Sistema } \\
\text { Ibérico (NW- } \\
\text { SE) }\end{array}$} & 5 Minutos & Lineal & 0,03 & $\mathrm{NO}(0,145)$ & $-1,845 \mathrm{E}-4$ & $\mathrm{NO}(0,799)$ & 0,003 \\
\hline & 15 Minutos & Lineal & 0,17 & SÍ $(0,032)$ & $-1,676 \mathrm{E}-4$ & NO $(0,291)$ & $-0,009$ \\
\hline & 30 Minutos & Lineal & 0,22 & $\mathrm{NO}(0,134)$ & $-8,388 \mathrm{E}-5$ & SÍ $(0,023)$ & $-0,014$ \\
\hline & 1 Hora & Lineal & 0,20 & $\mathrm{NO}(0,138)$ & $-6,395 \mathrm{E}-5$ & SÍ $(0,032)$ & $-0,010$ \\
\hline & 6 Horas & Semi-log. & 0,48 & SÍ $(0,000)$ & $-4,45 \mathrm{E}-6$ & SÍ $(0,045)$ & $-1,980 \mathrm{E}-4$ \\
\hline & 12 Horas & Semi-log & 0,52 & Sí $(0,000)$ & $-6,140 \mathrm{E}-6$ & $\mathrm{NO}(0,339)$ & $-9,771 \mathrm{E}-5$ \\
\hline & 24 Horas & Semi-log & 0,53 & SÍ $(0,000)$ & $-8,146 \mathrm{E}-6$ & NO $(0,423)$ & $8,964 \mathrm{E}-5$ \\
\hline \multirow{7}{*}{$\begin{array}{l}\text { Valle medio } \\
\text { y bajo del } \\
\text { Río Júcar } \\
\text { (W-E) }\end{array}$} & 5 Minutos & Semi-log & 0,08 & NO $(0,199)$ & $-1,912 \mathrm{E}-6$ & NO $(0,924)$ & $-1,873 \mathrm{E}-5$ \\
\hline & 15 Minutos & Semi-log & 0,08 & $\mathrm{NO}(0,330)$ & $-1,386 \mathrm{E}-6$ & $\mathrm{NO}(0,611)$ & \begin{tabular}{|l}
$-9,653 \mathrm{E}-5$ \\
\end{tabular} \\
\hline & 30 Minutos & Semi-log & 0,23 & SÍ $(0,045)$ & $-3,261 \mathrm{E}-6$ & $\mathrm{NO}(0,547)$ & $-1,273 \mathrm{E}-4$ \\
\hline & 1 Hora & Semi-log & 0,34 & Sí $(0,003)$ & \begin{tabular}{|l|}
$-6,271 \mathrm{E}-6$ \\
\end{tabular} & $\mathrm{NO}(0,983)$ & 5,271E-6 \\
\hline & 6 Horas & Semi-log & 0,56 & SÍ $(0,000)$ & $-7,181 \mathrm{E}-6$ & $\mathrm{NO}(0,360)$ & $-1,959 \mathrm{E}-4$ \\
\hline & 12 Horas & Semi-log & 0,61 & SÍ $(0,000)$ & $-9,215 \mathrm{E}-6$ & NO $(0,927)$ & $-1,971 \mathrm{E}-5$ \\
\hline & 24 Horas & Semi-log & 0,63 & SÍ $(0,000)$ & $-8,245 \mathrm{E}-6$ & NO $(0,405)$ & $-1,685 \mathrm{E}-4$ \\
\hline \multirow{7}{*}{$\begin{array}{l}\text { Sistema } \\
\text { Bético, } \\
\text { exposición } \\
\mathrm{NE}\end{array}$} & 5 Minutos & Semi- $\log$ & 0,35 & $\mathrm{NO}(0,248)$ & $-1,78 \mathrm{E}-5$ & NO $(0,615)$ & $-2,273 \mathrm{E}-4$ \\
\hline & 15 Minutos & Semi-log & 0,44 & NO $(0,779)$ & $-1,528 \mathrm{E}-5$ & $\mathrm{NO}(0,539)$ & $-2,018 \mathrm{E}-4$ \\
\hline & 30 Minutos & Semi-log & 0,56 & SÍ $(0,044)$ & $-2,601 \mathrm{E}-5$ & $\mathrm{NO}(0,832)$ & $-7,333 \mathrm{E}-5$ \\
\hline & 1 Hora & Semi-log & 0,69 & Sí $(0,024)$ & $-3,151 \mathrm{E}-5$ & $\mathrm{NO}(0,472)$ & $-2,632 \mathrm{E}-4$ \\
\hline & 6 Horas & Semi-log & 0,73 & SÍ $(0,010)$ & $-4,867 \mathrm{E}-5$ & $\mathrm{NO}(0,645)$ & $-2,151 \mathrm{E}-4$ \\
\hline & 12 Horas & Semi-log & 0,71 & SÍ $(0,014)$ & $-4,387 \mathrm{E}-5$ & NO $(0,639)$ & $-2,860 \mathrm{E}-4$ \\
\hline & 24 Horas & Semi-log & 0,76 & SÍ $(0,008)$ & $-3,752 \mathrm{E}-5$ & $\mathrm{NO}(0,613)$ & $-1,750 \mathrm{E}-4$ \\
\hline \multirow{7}{*}{$\begin{array}{l}\text { Sistema } \\
\text { Bético, } \\
\text { exposición } \\
\text { SE }\end{array}$} & 5 Minutos & Lineal & 0,47 & NO $(0,054)$ & 0,002 & NO $(0,091)$ & $-0,091$ \\
\hline & 15 Minutos & Lineal & 0,30 & NO $(0,621)$ & $4,731 \mathrm{E}-4$ & NO $(0,906)$ & $-0,005$ \\
\hline & 30 Minutos & Lineal & 0,05 & NO $(0,601)$ & 3,768E-4 & NO $(0,913)$ & $-0,004$ \\
\hline & 1 Hora & Lineal & 0,05 & NO $(0,681)$ & $2,041 \mathrm{E}-4$ & NO $(0,767)$ & $-0,007$ \\
\hline & 6 Horas & Lineal & 0,16 & $\mathrm{NO}(0,590)$ & $-1,136 \mathrm{E}-4$ & NO $(0,871)$ & 0,002 \\
\hline & 12 Horas & Lineal & 0,35 & $\mathrm{NO}(0,422)$ & $-1,196 \mathrm{E}-4$ & NO $(0,418)$ & 0,006 \\
\hline & 24 Horas & Lineal & 0,41 & NO $(0,358)$ & $-8,827 \mathrm{E}-5$ & NO $(0,257)$ & 0,005 \\
\hline
\end{tabular}


Por lo que respecta al criterio basado en las grandes estructuras del relieve (tabla 5) observamos cómo en el dominio de las sierras de dirección ibérica destaca la influencia lineal de la «altitud» para intervalos de tiempo intermedios (comprendidos entre 30 min. y 6 h), mientras que la «distancia al mar», que no interviene en los intervalos menores, se vuelve muy significativa, mediante relaciones semi-logarítmicas, a partir de las 6 h., llegando a explicar el $53 \%$ de los casos en el intervalo de $24 \mathrm{~h}$.

En el dominio de exposición E, correspondiente al valle medio-bajo del Río Júcar, la altitud no es significativa para ningún intervalo temporal, debido a que es una zona bastante plana. Aquí la variable significativa, con una relación de tipo semi-logarítmica, es la «distancia la mar», cuya influencia aumenta desde los 30 min hasta las 24 horas, en que llega a explicar el $63 \%$ de los casos.

Respecto al espacio dominado por las sierras béticas, diferenciábamos dos escenarios, el de exposición NE y el de exposición SE. El comportamiento del primero (NE) es similar al escenario anterior del valle medio-bajo del Júcar (componente E), ya que la variable significativa entre los $30 \mathrm{~min}$. y las 24 horas es la «distancia al mar», con coeficientes de determinación que superan el valor de 0,5 , llegando a explicar el 76\% de los casos en el intervalo de 24 h. En el escenario de exposición SE ninguna de las variables geográficas analizadas presenta significación.

\section{DISCUSIÓN Y CONCLUSIONES}

En los ambientes mediterráneos la mayor parte de los procesos naturales relacionados con la intensidad de lluvia ocurren en escalas de tiempo inferiores a la diaria. En este trabajo se ha llevado a cabo un estudio de intensidades máximas de lluvia registradas a intervalos de tiempo que varían entre los cinco minutos y las 24 horas. Lo primero que pone de manifiesto el análisis es que las intensidades máximas presentan una estructura diferente en función de la escala temporal de observación. En esta tendencia general destacan dos importantes puntos de inflexión, en $1 \mathrm{~h}$ y $6 \mathrm{~h}$, que diferencian tres fases en el comportamiento de las intensidades máximas: a) entre 5 min y 1 hora; b) entre 1 hora y 6 horas; y c) entre 6 horas y 24 horas. Estas fases mantienen rasgos de un comportamiento específico en la mayor parte de los análisis realizados. Así, por ejemplo, las intensidades máximas comprendidas entre los intervalos de 5 min y 1 hora muestran distribuciones normales, simétricas y mesocúrticas; mientras que entre las 6 horas y las 24 horas las distribuciones no son normales, presentan asimetría positiva y son leptocúrticas.

Este aspecto podría estar relacionado con la estructura de los campos de intensidad de lluvia descritos por Waymire y Gupta (1981) y Waymire et al. (1984). Según estos autores el patrón espacial de la lluvia muestra una estructura jerárquica que puede clasificarse en función de su extensión espacial, duración e intensidad, en cuatro categorías: a) synoptic áreas asociadas a tormentas ciclónicas (con extensión superior a los $10^{4} \mathrm{~km}^{2}$, más de 12 horas de duración e intensidades de $0,5 \mathrm{~mm} / \mathrm{h}$ ); b) large mesoscale precipitation areas (LMSA) también denominadas frontal rain areas o rainbands, asociadas a frentes (con superficies entre $10^{3}$ y $10^{4} \mathrm{~km}^{2}$, varias horas de duración e intensidades de aproximadamente $4 \mathrm{~mm} / \mathrm{h}$ ); c) small mesoscale precipitation areas (SMSA), formadas por un conjunto de células activas, cada una en una fase de desarrollo diferente (entre $10^{3}$ y $10^{2} \mathrm{~km}^{2}$ de extensión, varias horas 
de duración e intensidades en torno a los $8 \mathrm{~mm} / \mathrm{h}$ ) y c) células convectivas (menos de $10 \mathrm{~km}^{2}$, con duración inferior a 1 hora e intensidades por encima de los $100 \mathrm{~mm} / \mathrm{h}$ ).

Según este modelo las características que presentan las distribuciones de las intensidades máximas registradas entre 5 min y 1 hora reflejarían, sobre todo, la acción de potentes células convectivas, que precipitan grandes cantidades de agua en muy poco tiempo y sobre un espacio muy reducido. En este sentido, el comportamiento de las mismas es bastante similar y por ello las distribuciones presentan una disposición normal, entorno a la media. A partir de las 6 horas se reflejaría un comportamiento mixto, y por lo tanto con una distribución de intensidades más diferenciada y asimétrica, que recogería, además de algunas células convectivas de menor intensidad, las lluvias ocurridas en el seno de las LMSA y de las synoptic areas. Las intensidades registradas en a la escala temporal intermedia, entre 1 hora y 6 horas, responderían a las denominadas small mesoscale precipitation areas (SMSA).

Las diferentes escalas de este patrón, y en particular las células convectivas, están muy influenciadas por la distancia al mar y por el relieve. Por una parte la cercanía al mar implica la disponibilidad de un aporte continuo de humedad y, por otra parte, el incremento de altitud supone un efecto de disparo orográfico que fuerza al ascenso a los flujos de aire inestables, favoreciendo la precipitación.

También en el tipo de influencia que ejercen estos factores geográficos sobre las intensidades máximas se reflejan diferentes pautas de comportamiento en función del intervalo temporal de observación. Así, en general, la distancia al mar resulta significativa para todas las escalas de observación (a excepción de los 5 min., sujeta a factores muy locales), aunque comienza a tener importancia como factor explicativo a partir de las 6 horas, en que la cercanía a la fuente de aprovisionamiento de humedad en el aire resulta fundamental para alcanzar las intensidades máximas, sobre todo para una duración de 24 horas. La altitud, por su parte, sólo resulta significativa entre 30 min. y 6 horas, debido a que el efecto mecánico que ejercen los relieves favorece la condensación por un descenso de la temperatura del aire, pero no incrementa la presión parcial de vapor de agua.

Destaca también la clase de relación que se establece entre las variables, de tipo lineal para los intervalos entre 5 minutos y 1 hora y de tipo semilogarítmico a partir de las 6 horas. Este hecho podría tener relación con la complejidad de los procesos de precipitación. Hasta 1 hora de duración, el efecto de los factores geográficos es mucho más directo, ya que actúa fundamentalmente sobre células convectivas muy inestables que suelen entrar desde el mar, mientras que a partir de las 6 horas la altitud deja de resultar significativa y cobra importancia la distancia al mar y las características de los campos de lluvia de las escalas mayores (SMSA, LMSA y synoptic area), añadiéndose así complejidad a los procesos de precipitación.

Consecuencia de todo ello es el resultado del cluster que muestra dos patrones diferenciados de intensidad de lluvia que afecta a todas las escalas temporales de observación. El primero de ellos, ligado al efecto de disparo orográfico que ejercen las primeras alineaciones montañosas cercanas al mar, relaciona las intensidades máximas con rangos de altitud entre 200 y 400 metros y proximidad al mar $(20-30 \mathrm{~km})$. El segundo patrón asocia las lluvias menos intensas a mayores altitudes (900-1000 m) y distancias al mar (90-100 km), en relación con lluvias más generalizadas (LMSA o synoptic areas). Además, entre los intervalos temporales de cinco minutos y una hora los frentes de disparo orográfico de las intensidades 
máximas están por debajo de los 200 metros, mientras que a partir de la hora, se ubican entre 200 y 400 metros. Estos resultados son coherentes con los obtenidos por Millán et al. (2004) quienes, para el territorio valenciano entre 1959 y 2000, encuentran una tendencia diferente en la evolución de la precipitación (distinguiendo entre interior y costa) cuyo umbral estaría a unos $40 \mathrm{~km}$ de la costa, coincidiendo aproximadamente con los 400 m.s.n.m. de altitud. Según estos autores, mientras que las precipitaciones en el interior presentan una tendencia a reducirse, en la costa se ha registrado un incremento de precipitación como consecuencia de las situaciones meteorológicas de levante (backdoor cold fronts). Aunque el mencionado trabajo se basa en totales diarios y sería, por tanto, comparable únicamente con la escala de 24 horas, corrobora los resultados que se apuntan en el presente artículo.

Por lo que respecta al efecto que la diferente exposición del territorio puede ejercer sobre la influencia de los factores geográficos en las intensidades máximas, se trabajó sobre un criterio que distinguía entre escenarios de interior-litoral y sobre otro criterio que distinguía escenarios de configuración del territorio a partir de las grandes unidades estructurales del relieve. Los análisis llevados a cabo en los dos escenarios establecidos según el primer criterio (interior-litoral) no añaden información al análisis general. El segundo criterio, sin embargo, presenta cuatro escenarios (sierras ibéricas de dirección NW-SE; valle medio-bajo del río Júcar de dirección E, sierras béticas de exposición NE y sierras béticas de exposición $\mathrm{SE}$ ), algunos de los cuales suponen un incremento importante de coeficiente de determinación $\mathrm{r}^{2}$ de las variables geográficas como factor explicativo de las intensidades máximas. En este sentido destacan los escenarios de exposición a levante (valle medio-bajo del río Júcar de dirección E y sierras béticas de exposición NE) en los que la distancia al mar resulta determinante, sobre todo en los intervalos de tiempo mayores a 6 horas, llegando a explicar más del $70 \%$ de los casos. La variable altitud sólo es significativa (aunque con una $\mathrm{r}^{2}$ muy baja) en el escenario de sierras ibéricas de dirección NW-SE, entre 30 min y 1 hora.

Todo esto refuerza la idea, apuntada con anterioridad, de la importancia de la cercanía del mar como fuente de humedad para mantener las intensidades máximas en períodos superiores a 6 horas y el efecto de la altitud en intervalos menores a las 6 horas. Para el escenario de exposición asociado a las sierras béticas exposición SE ninguna variable a ninguna escala temporal resulta significativa. Este hecho confirma la importancia de la exposición del territorio, a barlovento o a sotavento de los vientos húmedos dominantes del NE, en la generación de las intensidades máximas, sobre todo en los intervalos de tiempo mayores, próximos a las 24 horas.

Como principales conclusiones del artículo se podrían apuntar algunas respuestas a las preguntas planteadas en la introducción y, a la vez, abrir nuevos interrogantes. A las cuestiones de qué escalas temporales de observación son las más representativas y cuál es su estructura, podríamos apuntar los intervalos de 5 minutos, 1 hora, 6 horas y 24 horas. Los intervalos entre 5 minutos y 1 hora representan los fenómenos relacionados con el tiempo de desarrollo de células convectivas y presentan distribuciones estadísticas normales, simétricas y mesocúrticas. Los intervalos entre 6 horas y 24 horas representan un cambio en el comportamiento de las intensidades máximas, con distribuciones no normales, asimétricas y leptocúrticas que podrían relacionarse con las características de una LMSA o una synoptic area. El período intermedio entre 1 hora y 6 horas comprendería un intervalo mixto, con características propias de una SMSA. 
Respecto a las cuestiones de qué factores geográficos condicionan los valores extremos de intensidad y si su papel difiere de unas escalas de observación a otras, destaca como factor más importante la distancia al mar que resulta significativa en casi todas las escalas temporales (exceptuando los $5 \mathrm{~min}$ ), si bien tiene un peso mayor a partir de las 6 horas y sobre los escenarios con una clara exposición E ó NE. La altitud, por su parte, sólo resulta significativa para intervalos comprendidos entre 30 min y 6 horas, sobre todo en el escenario de las sierras ibéricas. Destaca, además, el escenario de exposición de sierras béticas exposición SE que, situado a barlovento de los vientos dominantes, no resulta significativo en la explicación de las intensidades máximas.

Somos conscientes que este trabajo sólo es una primera aproximación al comportamiento de la lluvia según la escala temporal de observación y que serían necesarios estudios más detallados que permitieran diferenciar las intensidades máximas en función del tipo de situación meteorológica que las ha originado. Asimismo sería interesante poder incluir en el análisis más indicadores de lluvia como la persistencia o la irregularidad, cuyo comportamiento también resulta muy dependiente de la escala temporal de observación. No obstante creemos que el interés del trabajo radica en su carácter empírico, ya que se ha trabajado con datos reales de intensidades máximas registradas a diferentes intervalos de observación, en un entorno geográfico variado y heterogéneo y bajo unas condiciones climáticas mediterráneas.

\section{AGRADECIMIENTOS}

Este trabajo ha sido realizado en el marco del proyecto CGL2013-42728-R, financiado por el Ministerio de Ciencia e Innovación y cofinanciado por fondo FEDER.

Los autores asimismo desean mostrar su agradecimiento a la Confederación Hidrográfica del Júcar, en particular al SAIH (Sistema Automático de Información Hidrológica), por la cesión de los datos hidrológicos en los que se basa este estudio.

\section{BIBLIOGRAFÍA}

ALBENTOSA, L.M. (1989): «Las precipitaciones extraordinarias en Cataluña», en Avenidas fluviales e inundaciones en la cuenca del mediterráneo. Instituto Universitario de Geografía de la Universidad de Alicante y Caja de Ahorros del Mediterráneo, 51-66.

ALLAMANO, P.; CLAPS, F.; LAIO F. y THEA C. (2009): «A data-based assessment of the dependence of short-duration precipitation on elevation»; Physics and Chemistry of the Earth, 34, 635-641.

ARMENGOT, R. (2002): Las lluvias intensas en la Comunidad Valenciana, Ministerio de Medioambiente, Valencia, $263 \mathrm{p}$.

BACCHINI, M. y ZANNONI, A. (2003): «Relations between rainfall and triggering of debris-flow: case study of Cancia (Dolomites, Northeastern Italy)», Nat. Hazards Earth Syst. Sci., 3, 71-79.

BEGUERÍA, S.; VICENTE-SERRANO, S.; LÓPEZ-MORENO, J.I. y GARCÍA-RUÍZ J.M. (2009): «Annual and seasonal mapping of peak intensity, magnitude and duration of extreme precipitation events across a climatic gradient, northeast Spain»; Int. J. Climatol. 29, 1759-1779. 
BENGTSSON, L. y MILLOTI, S. (2010): «Extreme storms in Malmö, Sweden», Hydrological Processes, 24, 3462-3475.

BERNE, A.; DELRIEU, G. y BOUDEVILLAIN, B. (2009): «Variability of the spatial structure of intense Mediterranean precipitation»; Advances in water resources, 32, 1031-1042.

BONI, G.; PARODI, A. y SICCARDI, F. (2008): «A New Parsimonious Methodology of Mapping the Spatial Variability of Annual Maximum Rainfall in Mountainous Environments», Journal of Hydrometeorology, 9, no. 3: 492-506.

BONI, G; PARODI, A. y RUDARI, R. (2005): «Extreme rainfall events: Learning from raingauge time series»; Journal of Hydrology, 327, 304-314.

BRACKEN, L.J.; COX, N.J. y SHANNON, J. (2008): «The relationship between rainfall inputs and flood generation in south-east Spain», Hydrological Processes, 22, 683-696.

BRUNETTI, M.; COLACINO, M.; MAUGERI, M. y NANNI, T. (2001): «Trends in the daily intensity of precipitation in Italy from 1951 to 1996»; International Journal of Climatology, 21, 299-316.

BULL, L.J.; KIRKBY, M.J.; SHANNON, J. y HOOKE, J.M. (1999): «The impact of rainstorms on floods in ephemeral channels in southeast Spain»; Catena, 38, 191-209.

CAMARASA, A.M. y SEGURA (2001): «Flood events in Mediterranean ephemeral streams (ramblas) in Valencia region, Spain», Catena, 45, 229-249.

CAMARASA, A.M.; LÓPEZ-GARCÍA, M.J y SORIANO, J. (2010): «The effect of observation timescales on the characterisation of extreme Mediterranean precipitation»; $A d v$. Geosci., 26: 61-64.

CAMMERAAT, E. (2004): «Scale dependent thresholds in hydrological and erosion response of a semi-arid catchment in southeast Spain»; Agriculture, Ecosystems and Environment, 104, 317-332.

CANNON, S.H. (1988): «Regional rainfall-threshold conditions for abundant debris-flow activity»; en: Landslide, Floods and Marine effects of the storm of 3-5 January 1982 in the San Francisco Bay Region, California; (Eds) ellen S.D. and Wieczorek, G.F.; USGS Professional Paper 1434, 35-42.

CUDENNEC, C.; LEDUC, C. and KOUTSOYIANNIS, D. (2007): «Dryland hydrology in Mediterranean regions -a review», Hydrological Sciences Journal, 52 (6), 1077-1087.

CHIEN-YUAN, C.; TIEN-CHIEN, C.; FAN-CHIEH, Y; WEN-HUI, Y. y CHUN-CHIEH, T. (2005): «Rainfall duration and debris-flow initiated studies for real-time monitoring», Environ. Geol., 47, 15-724.

DUNKERLEY, D. (2008): «Identifying individual rain events from pluviograph records: a review with analysis of data from an Australian dryland site»; Hidrol. Process., 22, 50245036.

EGOZCUE, J.J.; PAWLOWSKY-GLAHN, V.; ORTEGO, M.I. y TOLOSANA-DELGADO, R. (2006): «The effect of scale in daily precipitation hazard assessment»; Nat. Hazards Earth Syst. Sci., 6, 459-470.

ESTRELA, T.; HIDALGO, A. y SULLANA, J. (2004): Júcar Pilot River Basin, Provisional Article 5 Report Pursuant to the Water Framework Directive; Confederación Hidrográfica del Júcar, Ministerio de Medio Ambiente, 208 pp, available at: www.chj.es, 2004.

FREI, C. and SCHÄR, C.: A precipitation climatology of the Alps from high-resolution raingauge observations. International Journal of Climatology; 18, 873-900; 1998. 
GIL OLCINA, A. (1989): «Causas climáticas de las riadas», en: Avenidas fluviales e inundaciones en la cuenca del mediterráneo; Instituto Universitario de Geografía de la Universidad de Alicante y Caja de Ahorros del Mediterráneo, 15-30.

GABRIELE, S. Y ARNELL, N. (1991): «A hierarchical approach to regional flood frequency analysis», Water Resources Research, vol. 27, n 6, pp 1281-1289.

HOMAR, V.; ROMERO, R.; RAMIS, C. y ALONSO, S. (2002): «Numerical study of the October 2000 torrential precipitation event over Spain: analysis of the synoptic-scale stationarity»; Annales Geophysicae, 20, 2047-2066.

HOUZE, R.; JAMES, C.N. y MEDINA, S. (2001): «Radar observations of precipitation and airflow on the Mediterranean side of the Alps: Autumn 1998 and 1999», Quart. J. Roy. Meteor. Soc., 127, 2537-2558.

JEBARI, S.; BERNDTSSON, R. UVO, C. y BAHRi, A. (2007): «Regionalizing fine timescale rainfall affected by tophography in semi-arid Tunisia»; Hydrological Sciences Journal, 52, 1199-1215.

KYSLEY, J.; PICEK, J. y HUTH, R. (2007): «Formation of homogeneous regions for regional frequency analysis of extreme precipitation events in the Czech Republic», Stud. Geophys. Geod., 51, pp 327-344.

KOKKONEN, T.; KOIVUSALO, H. KARVONEN, T., CROKE, B. and JAKEMAN, A. (2004): «Exploring streamflow response to effective rainfall across event magnitude scale»; Hydrol. Process. 18, 1467-1486.

LANGE, J.; GREENBAUM, N.; HUSARY, S.; GHANEM, M.; LEIBUNDGUT, C. y SHICK, A.P. (2003): « Runoff generation from sucessive simulated rainfalls on a rocky, semi-arid, Mediterranean hillslope», Hydrological Processes, 17, 279-296.

LARSON, WE; LINDSTROM, MJ y SCHUMACHER, TE (1997): «The role of severe storms in soil erosion: a problem needing consideration», Journal of Soil and Water Conservation, 52, 90-95.

LLASAT, M.C. (2001): «An objetive classificationof rainfall events on the basis of their convective features: application to rainfall intensity in the northeast of Spain»; Int. J. Climatol, 21, 1385-1400.

MARTINEZ-MENA, M.; CASTILLO, V. y ALBALADEJO, J. (2001): «Hydrological and erosional respnse to natural rainfall in a semi-arid area of South-east Spain», Hydrologial Processes, 15, 557-571, doi: 10.1002/hyp.146.

MARTÍN-VIDE, J. (1987): Característiques climatológiques de la precipitació en la franja costera maditerrània de la Península Ibèrica; Institut Cartogràfic de Catalunya, Barcelona, 245 p.

MARTÍN-VIDE, J. (2004): «Spatial distribution of daily precipitation concentration index in peninsular Spain», International Journal of Climatology, 24, 959-971.

MILLÁN, M.; ESTRELA, J.J. y CASELLES, V. (1995): «Torrencial precipitations on the Spanish east coast: The role of the Mediterranean surface temperature»; Atmospheric Research, 36, 1-16.

MILLÁN, M.; ESTRELA, J.J. y MIRÓ, J. (2004): «Rainfall components: variability and spatial distribution in a Mediterranean área (Valencia Region)», Journal of Climate, 18, 2682-2705.

MONTESARCHIO, V.; LOMBARDO, F. y NAPOLITANO, F. (2009): «Rainfall thresholds and flood warning: an operative case study», Nat. Hazards Earth Syst. Sci., 9, 135-144. 
PEÑARROCHA, D.; ESTRELA, MJ. y MILLÁN, M. (2002): «Classification of daily rainfall patterns in a Mediterranean area with extreme intensity levels: the Valencia region»; Int. J. Climatol, 22, 677- 695. DOI: 10.1002/joc.747.

POESEN J. y BUNTE, K. (1996): «The effects of rock fragments on desertification processes in Mediterranean environments», en: Mediterranean Desertification and Land Use, Brandt CJ, Thornes JB (eds), Wiley: Chichester, 247-269.

QUEREDA SALA, J. (1989): «Las interacciones atmósfera-océano en la climatología del Mediterráneo occidental»; en Avenidas fluviales e inundaciones en la cuenca del mediterráneo; Instituto Universitario de Geografía de la Universidad de Alicante y Caja de Ahorros del Mediterráneo, 67-88.

ROE, G.H. (2005): «Orographic precipitation», Annu. Rev. Earth Planet. Sci.; 33, 645-671.

ROMERO, R.; RAMIS, C. y GUIJARRO, J.A. (1999): «Daily rainfall patterns in the Spanish Mediterranean area: an objective classification»; Int. J. Climatol, 19, 95-112.

RUSJAN, S.; KOBOLD, M. y MIKOs, M. (2009): «Characteristicas of the extreme rainfall event and consequent flash floods in W Slovenia in September 2007»; Nat. Hazards Earth Syst. Sci., 9, 947-956, 2009.

SALSON-CASADO, S. y GARCÍA-BARTUAL, R. (1998): «Desagregación de lluvias para aplicaciones en simulación de sistemas de recursos hidráulicos»; Revista de Obras Públicas, $\mathrm{n}^{\mathrm{o}} 3.3 .78,25-35$.

VALDÉS, J.B.; RODRIGUEZ-ITURBE, I. y GUPTA, V.K. (1985): «Approximations of temporal rainfall from a multidimensional model», Water Resources Research, vol 21, 8, 1259-1270.

WAYMIRE, E. y GUPTA, V.K. (1981): «The mathematical structure of rainfall representations 1. A review of the stochastic rainfall models»; Water. Resour. Reser. 17 (5), 1261 1272.

WAYMIRE, E.; GUPTA, V.K. y RODRÍGUEZ-ITURBE, I. (1984): «A spectral theory of rainfall intensity at the Meso-B scale»; Water Resources Research, vo. 20, n 10, 14531465.

WILSON, R.C. y WIECZOREK, G.F. (1995): «Rainfall thresholds for the initation of debris flow at La Honda, California», Environmental Engineering and Geoscience, 1 (1), 11-27.

YAIR, A. y LAVEE, H. (1985): «Runoff generation in arid and semi-arid zones»; en: Anderson and Burt (Eds.), Hydrological Forecasting, 183-220.

YAIR, A. y RAZ-YASSIF, N. (2004): «Hydrological processes in a small arid carchment: scale effects of rainfall and slope length»; Geomorphology, 61, 155-169.

ZEHE, E. y SIVAPALÁN, M. (2009): «Threshold behviour in hydrological systems as (human) geo-siystems: manifestations, controls, Implications», Hydrological Earth System Sciences, 13, 1273-1297.

ZIV, B.; SAARONI, H. y ALPERT, P. (2004): «The factors governing the summer regime of the eastern Mediterranean»; Int. J. Climatol. 24, 1859-1871; doi: 10.1002/joc.1113. 